Pacific Journal of Mathematics

DIHEDRAL GROUP ACTIONS ON HOMOTOPY COMPLEX 


\title{
DIHEDRAL GROUP ACTIONS ON HOMOTOPY COMPLEX PROJECTIVE THREE SPACES
}

\section{Mark Hughes}

\begin{abstract}
Let $\mathbf{D}_{2 m}$ be the dihedral group of order $2 \mathrm{~m}$. Given an odd prime $m$ such that the projective class group of $\mathbf{D}_{2 m}$ has 2-rank $=0$, we construct smooth $\mathbf{D}_{2 m}$-actions on an infinite number of pairwise non-diffeomorphic (distinguished by Pontryagin class) manifolds each of which is homotopy equivalent to $\mathrm{C} P^{3}$. This is accomplished by applying equivariant surgery theory to normal maps created by an equivariant transversality argument.
\end{abstract}

1. Introduction. The question which we deal with here is: "Which finite groups can act on differentiably non-standard homotopy $\mathbf{C} P^{3}$ 's?" We use equivariant surgery theory to construct dihedral group actions on an infinite number of differentiably distinct smooth manifolds which are homotopy equivalent to $\mathbf{C} P^{3}$.

According to [MY], there is a one-to-one correspondence between the integers and the set (actually, it is a group) of diffeomorphism classes of 6-dimensional, smooth, closed manifolds which are homotopy equivalent to $\mathrm{CP}^{3}$ (such manifolds shall hereafter be called homotopy $\mathbf{C} P^{3}$ 's). For every integer $k$, there is a unique homotopy $C P^{3}$, denoted $X_{k}$, with first Pontryagin class $P_{1}\left(X_{k}\right)=(4+24 k) x^{2}$, where $x \in H^{2}\left(X_{k}\right)$ is a generator. Then, $X_{0}$ is the standard $\mathbf{C} P^{3}$. All actions shall be effective and smooth.

Some information is known about smooth finite group actions on homotopy $\mathrm{CP}^{3}$ 's. For instance, infinitely many homotopy $\mathrm{CP}^{3}$ 's admit a $\mathbf{Z}_{m}$-action for almost every prime number $m$. (For this, and other interesting results, we refer the reader to [DM].) On the other hand, in [M1], it is shown that if $X_{k}$ admits a smooth, effective $\mathbf{Z}_{m} \times \mathbf{Z}_{m} \times \mathbf{Z}_{m}$-action, for any odd prime $m$, then $k=0$, i.e., $X_{k}=\mathbf{C} P^{3}$. (There is a more restricted version of this result for $m=2$. For information about involutions on homotopy $\mathbf{C} P^{3}$ 's, we refer the reader to [DMS].)

In this paper, we shall consider the case of dihedral group actions. To my knowledge, these are the first examples of non-abelian group 
actions on non-standard $\mathbf{C} P^{3}$. Specifically, our main result is:

THEOREM A. Let $\mathbf{D}_{2 m}$ be the dihedral group of order $2 m$, where $m$ is an odd prime. If the projective class group $\widetilde{K}_{0}\left(\mathbf{Z}\left[\mathbf{D}_{2 m}\right]\right)$ has 2-rank= 0 , then there are infinitely many integers $k$ for which $X_{k}$ admits $a$ $\mathbf{D}_{2 m}$-action. In particular, this holds for $m \leq 67$.

The main tool used to prove the above theorems is equivariant surgery theory (see $[\mathbf{D P}]$ and $[\mathbf{P R}]$ ). The features of this theory which are relevant to our work shall be outlined in the next section. The third section provides the proof of Theorem A. At this point, I would like to thank Professor Heiner Dovermann for his help and encouragement. Thanks also goes to the Florida State University Council for Research and Creativity which provided financial support during the time this work was undertaken. Finally, I thank the referee for many helpful comments.

2. Background. Let $G$ be a finite group. Equivariant $(G-)$ surgery is a process for constructing $G$-manifolds which are $G$-homotopy equivalent to a given $G$-manifold $Y$. (A homotopy $F: X \times I \rightarrow Y$ is a $G$-homotopy if $F(\cdot, t)$ is a $G$-map for all $t$.) Two major steps are involved in this process.

1. We build a $G$-normal map $(X, f, b)$ with target manifold $Y$. This can be thought of as an approximation to a $G$-homotopy equivalence.

2. We must determine whether or not the obstructions to performing $G$-surgery to a $G$-homotopy equivalence vanish. The process of $G$-surgery converts $X$ to a $G$-manifold $X^{\prime}$ and $f$ to a $G$-map $f^{\prime}: X^{\prime} \rightarrow Y$ which is a $G$-homotopy equivalence.

Before we elaborate on this, we need some definitions.

Definition 2.1. A $G$-manifold is said to satisfy the gap hypothesis if given a nontrivial subgroup $H \subseteq G$ and a component $F$ of $X^{H}$, we have $2 \operatorname{dim} F<\operatorname{dim} X$.

(Other definitions of the gap hypothesis are possible, however this is the one which suits our needs.)

Recall that a smooth $G$-vector bundle is a triple $(E, p, B)$, where $p: E \rightarrow B$ is an ordinary smooth vector bundle such that $E$ and $B$ support smooth $G$-actions and the projection $p$ is a $G$-map. We also require that given $g \in G$ and $b \in B$, the map restricted to fiber $g: E_{b} \rightarrow E_{g(b)}$ is linear. 
At this point, for simplicity, instead of defining $G$-normal maps, we choose to define a special type of $G$-normal map, the notion of an adjusted $G$-normal map. (See [D1]. The notion of a $G$-normal map can be found, for example, in [H] or [PR].)

Definition 2.2. An adjusted $G$-normal map with target $Y$ is a triple $(X, f, b)$, where

(1) $X$ is a smooth, oriented, closed $G$-manifold which satisfies the gap hypothesis and is of dimension $\geq 5$. $Y$ is a smooth, oriented, closed $G$-manifold which is simply connected and of the same dimension as $X$.

(2) $f: X \rightarrow Y$ is a smooth, degree $1 G$-map which induces a $G$ homotopy equivalence between the singular sets $X^{s}$ and $Y^{s}$. (Recall that $X^{s}=\left\{x \in X: G_{x} \neq 1\right\}$.)

(3) $b$ is a stable $G$-vector bundle isomorphism between $T X \oplus$ $f^{*}\left(\eta_{-}\right)$and $f^{*}\left(T Y \oplus \eta_{+}\right)$, for some pair of $G$-vector bundles $\eta_{ \pm}$. That is, there exists a $G$-representation $V$ such that $b$ is a $G$ vector bundle isomorphism between $T X \oplus f^{*}\left(\eta_{-}\right) \oplus(X \times V)$ and $f^{*}\left(T Y \oplus \eta_{+}\right) \oplus(X \times V)$.

We have a further important definition.

Definition 2.3. Let $\eta_{+}$and $\eta_{-}$be $G$-vector bundles over a $G$ manifold $Y$. Assume that given $H \subseteq G$ and $y \in Y^{H}$, we have $\operatorname{dim}\left(\left.\eta_{+}\right|_{y}\right)^{H}=\operatorname{dim}\left(\left.\eta_{-}\right|_{y}\right)^{H}$. Then $\omega: \eta_{+} \rightarrow \eta_{-}$is a $G$-fiber homotopy equivalence if it is a proper, fiber preserving $G$-map such that, given $H \subseteq G$ and $y \in Y^{H}$, the map $\left(\left.\omega\right|_{y}\right)^{H}:\left(\left.\eta_{+}\right|_{y}\right)^{H} \rightarrow\left(\left.\eta_{-}\right|_{y}\right)^{H}$ has degree 1 when extended to one point compactifications.

Using ideas found in $\S 11$ of Chapter 3 in [PR], an adjusted $G$ normal map can be constructed from a $G$-fiber homotopy equivalence over $Y$ provided that certain conditions are met. This shall be carried out in $\S 3$ of this paper.

Once our adjusted $G$-normal map is constructed, we proceed to step 2 , which is to determine whether surgery to a $G$-homotopy equivalence is possible.

We first mention that an equivariant map $f: X \rightarrow Y$ is a $G$ homotopy equivalence if and only if $f^{H}: X^{H} \rightarrow Y^{H}$ is an ordinary homotopy equivalence for all $H \subseteq G$. (See [B].) Therefore, given our adjusted $G$-normal map $(X, f, b)$, we must convert $X$ to a $G$ manifold $X_{\eta}$ and $f$ to a $G$-map $F: X_{\eta} \rightarrow Y$ such that $F^{H}$ is a homotopy equivalence for all $H \subseteq G$. There is a surgery obstruction to achieving this as indicated in the following proposition. At this point, 
we make the assumption that the dimension of $Y$ is even and that $G$ contains no elements $g$ of order 2 with $2\left(\operatorname{dim}\left(Y_{\alpha}^{H}\right)^{g}+1\right)=\operatorname{dim} Y_{\alpha}^{H}$. This will ensure that the following obstruction is well-defined. Our constructions will fall within this constraint. For more on this point, see [Mo] and $\S 3$ of [D].

Proposition 2.4. Let $(X, f, b)$ be an adjusted $G$-normal map with target $Y$. There is an obstruction $\sigma_{1}(f, b)$ which vanishes if and only if $(X, f, b)$ is $G$-normally cobordant to an adjusted $G$-normal map $\left(X_{\eta}, F, B\right)$, where $F: X_{\eta} \rightarrow Y$ is a G-homotopy equivalence.

That is, $\sigma_{1}(f, b)$ vanishes if and only if $G$-surgery can be used to convert $X$ to $X_{\eta}$ and $f$ to a $G$-homotopy equivalence $F: X_{\eta} \rightarrow Y$. The proof of this proposition may be found in [D]. (See Corollary 1.1 on p. 853.) Related results involving $G$-normal maps are wellknown and can be found in [PR] and [DR2]. Also, see [BQ]. We note that this surgery is done relative to the singular set $X^{s}$. The obstruction $\sigma_{1}(f, b)$ is an element of the Wall group $L_{n}^{h}(\mathbf{Z}[G], w)$, where $n=\operatorname{dim} Y$ and $w: G \rightarrow \mathbf{Z}_{2}$ is the orientation homomorphism of the $G$-action on $Y$.

It is often easier to deal with $L_{n}^{s}(\mathbf{Z}[G], w)$, the surgery obstruction group for simple homotopy equivalences instead of $L_{n}^{h}(\mathbf{Z}[G], w)$. These two groups are related by the Rothenberg exact sequence [Sh]:

$$
\cdots \rightarrow L_{n}^{s}(\mathbf{Z}[G], w) \rightarrow L_{n}^{h}(\mathbf{Z}[G], w) \stackrel{\alpha_{G}}{\rightarrow} H^{n}\left(\mathbf{Z}_{2} ; \mathrm{Wh}(G)\right) \rightarrow \cdots,
$$

where $\mathrm{Wh}(G)$ is the Whitehead group of $G$ and $\alpha_{G}$ is the torsion homomorphism to be considered shortly. The Tate cohomology group $H^{n}\left(\mathbf{Z}_{2} ; \mathrm{Wh}(G)\right)$ is defined as:

$$
\left\{\delta \in \mathrm{Wh}(G): \delta=(-1)^{n} \delta^{*}\right\} /\left\{\tau+(-1)^{n} \tau^{*}: \tau \in \mathrm{Wh}(G)\right\},
$$

where $*$ denotes the conjugation involution based on the orientation homomorphism $w$.

Let's suppose that our adjusted $G$-normal map $(X, f, b)$ with target $Y$ has been constructed from a $G$-fiber homotopy equivalence $\omega: \eta_{+} \rightarrow \eta_{-}$over $Y$. In this situation, the work of Dovermann ([D]) and Dovermann-Rothenberg ([DR1]) can be applied to give us information on $\alpha_{G}\left(\sigma_{1}(f, b)\right) \in H^{n}\left(\mathbf{Z}_{2} ; \mathrm{Wh}(G)\right)$. Given a $G$-fiber homotopy equivalence $\omega$, its generalized Whitehead torsion $\tau(\omega)$ can be defined as an element of the generalized Whitehead group $\widetilde{\mathrm{Wh}}(G)=\bigoplus_{(H) \subseteq G} \mathrm{Wh}\left(N_{G}(H) / H\right)$, where $(H)$ denotes the conjugacy 
class of $H$. Let $T$ denote the conjugation on $\widetilde{\mathrm{Wh}}(G)$ and [.] denote the cohomology class as indicated above.

LEMMA 2.5. Let $(X, f, b)$ be an adjusted G-normal map constructed as above such that $f^{H}$ is an $N_{G}(H) / H$-diffeomorphism for each nontrivial subgroup $H$ of $G$. Then $\alpha_{G}\left(\sigma_{1}(f, b)\right)=[T \tau(\omega)]$.

Proof. A formula for $\alpha_{G}\left(\sigma_{1}(f, b)\right)$ can be given by combining Theorems A and B along with Proposition 6.6 of Dovermann's paper [D]. Noting that our assumption on $f$ implies that $\tau\left(f^{s}\right)=0$, the formula reduces to $\alpha_{G}\left(\sigma_{1}(f, b)\right)=[T \tau(\varphi)]$, where $\varphi$ is a certain $G$ fiber homotopy equivalence associated with the adjusted $G$-normal map $(X, f, b)$. As can be seen from $\S 6$ of [D], $\varphi$ is closely related to our $G$-fiber homotopy equivalence $\omega$. Indeed, by stabilizing with an appropriate complex $G$-bundle, we can arrive at the same $G$-fiber homotopy equivalence. Then, the addition formula of DovermannRothenberg (Corollary 8.15 of [DR1]) implies that $\tau(\varphi)=\tau(\omega)$ which finishes our proof. (We mention that the results of [D] and [DR1] are written in terms of sphere bundles. The Whitney sum corresponds to fiberwise join. This is not a restriction for us. See $\S \S 1-13$ of [PR].)

LemMa 2.6. Suppose that $\omega: \eta_{+} \rightarrow \eta_{-}$is a G-fiber homotopy equivalence over an even dimensional G-manifold $Y$. Let $\tilde{\omega}=\omega \oplus \omega: \eta_{+} \oplus$ $\eta_{+} \rightarrow \eta_{-} \oplus \eta_{-}$. If the ordinary Whitehead group $\mathrm{Wh}(G)$ is torsion free and if $\tau(\tilde{\omega})$ has non-zero coordinate only in $\mathrm{Wh}(G)$, then $[T \tau(\tilde{\omega})]=0$.

Proof. In [DR1], a formula for the generalized Whitehead torsion of a $G$-fiber homotopy equivalence is given. From Corollary 8.15 of that paper, it follows that with our set-up, $\tau(\tilde{\omega})$ is twice an element of $\widehat{\mathrm{Wh}}(G)$. Therefore, $T \tau(\tilde{\omega})$ is also a "multiple of 2 ". In general, this is not enough to show that $[T \tau(\tilde{\omega})]$ vanishes in

$$
H^{n}\left(\mathbf{Z}_{2} ; \mathrm{Wh}(G)\right)=\left\{\delta \in \mathrm{Wh}(G): \delta=\delta^{*}\right\} /\left\{\tau+\tau^{*}: \tau \in \mathrm{Wh}(G)\right\} .
$$

(Note that we are assuming $n=\operatorname{dim} Y$ is even.)

However, with our additional assumption that $\mathrm{Wh}(G)$ is torsion free, the result follows.

Indeed, $T \tau(\tilde{\omega})$ lies in $\left\{\delta \in \mathrm{Wh}(G): \delta=\delta^{*}\right\}$ and as explained above, $T \tau(\tilde{\omega})=2 x$, for some $x \in \mathrm{Wh}(G)$. We claim that we must have $x \in\left\{\delta \in \mathrm{Wh}(G): \delta=\delta^{*}\right\}$. Suppose that $\alpha \in \mathrm{Wh}(G)=\bigoplus \mathbf{Z}$ and let $\varphi$ be any homomorphism from $\mathrm{Wh}(G)$ to itself (in particular, $\varphi=$ $*$ ). Since $\varphi$ is a homomorphism and $\operatorname{Wh}(G)$ is torsion free, it follows 
easily that $\varphi(2 \alpha)=2 \alpha \Rightarrow \varphi(\alpha)=\alpha$. Therefore, we have $T \tau(\tilde{\omega})=$ $2 x=x+x=x+x^{*}$ and hence $[T \tau(\tilde{\omega})]=0$ in $H^{n}\left(\mathbf{Z}_{2} ; \mathrm{Wh}(G)\right)$ as desired.

We note that we shall be working with the group $\mathbf{D}_{2 m}$, where $m$ is an odd prime. (The conjugation involution on $\operatorname{Wh}\left(\mathbf{D}_{2 m}\right)$ will be defined in terms of the nontrivial orientation homomorphism $w$, i.e., elements of order 2 shall reverse orientation and be sent to -1 .) It is shown in [0] that $S K_{1}\left(\mathbf{Z}\left[\mathbf{D}_{2 m}\right]\right)$, the torsion part of $\mathrm{Wh}\left(\mathbf{D}_{2 m}\right)$, vanishes for all odd primes $m$. (Also see [Ma].) We also note that $\tau(\tilde{\omega})$ will have non-zero coordinate only in $\mathrm{Wh}(G)$ in our geometric set-up. (See Lemma 2.5.)

Our purpose for introducing the Rothenberg sequence is to show that $\sigma_{1}(f, b) \in L_{n}^{h}(\mathbf{Z}[G], w)$ comes from an element, $\sigma_{1}^{s}(f, b) \in$ $L_{n}^{s}(\mathbf{Z}[G], w)$, which will be shown to vanish, thereby guaranteeing that $\sigma_{1}(f, b)=0$, and that surgery to a $G$-homotopy equivalence is possible. Clearly, $\sigma_{1}(f, b)$ will come from some $\sigma_{1}^{s}(f, b)$ if $\alpha_{G}\left(\sigma_{1}(f, b)\right)=0$.

\section{Proof of Theorem A. In this section, we shall give the proof of:}

TheOREM A. Let $\mathbf{D}_{2 m}$ denote the dihedral group of order $2 m$, where $m$ is an odd prime. If the projective class group $\widetilde{K}_{0}\left(\mathbf{Z}\left[\mathbf{D}_{2 m}\right]\right)$ has 2-rank $=0$, then there are infinitely many $k$ for which $X_{k}$ admits $a$ $\mathbf{D}_{2 m}$-action. More precisely, given $p$ and $q$, relatively prime integers each $\equiv 1 \bmod m, X_{k}$ admits a $\mathbf{D}_{2 m}$-action, where $k=$ $\left(p^{2}-1\right)\left(q^{2}-1\right) / 3$.

Let us first make some remarks about $\widetilde{K}_{0}\left(\mathbf{Z}\left[\mathbf{D}_{2 m}\right]\right)$. It is known that $\widetilde{K}_{0}\left(\mathbf{Z}\left[\mathbf{D}_{2 m}\right]\right)=\Gamma$, the class group of the maximal real subfield of $\mathbf{Q}(\xi)$, where $\xi=e^{2 \pi i / m}$. (See [L].) As shall be indicated below, we are interested in knowing for which $m, \Gamma$ has 2 -rank $=0$. This is not known in general, however it is known that the class number $h^{+}$, which is the order of $\gamma$, vanishes for primes $m \leq 67$. (See p. 38 and $\S 3$ of the appendix in [Wa].)

Our proof will depend upon an appropriate choice of a model $Y$ on which to base our surgery constructions. We will then construct a $\mathbf{D}_{2 m}$-fiber homotopy equivalence over $Y$, and from it, an adjusted, $\mathbf{D}_{2 m}$-normal map. A key feature of our construction will be an additional amount of symmetry built into the normal map. Finally, we will show that our set-up is such that all obstructions to surgery vanish. By varying the parameters of our construction, we will obtain infinitely many $X_{k}$ as claimed. 
Our model $Y$ and $\mathbf{D}_{2 m}$-fiber homotopy equivalence will be constructed so as to satisfy an important technical condition stated below as Definition 3.1 which will allow us to build from them an adjusted $\mathbf{D}_{2 m}$-normal map.

First, we set up some notation. Let $G$ be finite. Given any irreducible, real $G$-representation $\psi$, we define $m_{\psi}: R O(G) \rightarrow \mathbf{Z}$ by setting $m_{\psi}(V)$ equal to the multiplicity of $\psi$ in the virtual representation $V .(R O(G)$ denotes the real representation ring of $G$. Let $d_{\psi}$ denote the dimension of the real division algebra of $\mathbf{R}$-linear $G$-endomorphisms of $\psi, \operatorname{Hom}_{\mathbf{R}}^{G}(\psi, \psi)$.

Definition 3.1 (Transversality Condition). Let $\omega: \eta_{+} \rightarrow \eta_{-}$be a $G$-fiber homotopy equivalence over the smooth $G$-manifold $Y$. For each $H \in \operatorname{Iso}(Y)=\left\{G_{y}: y \in Y\right\}$ and each component $Y_{\alpha}^{H} \subseteq Y^{H}$ the following holds. Let $y \in Y_{\alpha}^{H}$. For each real $H$-representation $\psi$ with $m_{\psi}(\eta-\mid y) \neq 0$ we have

$$
\operatorname{dim} Y_{\alpha}^{H}=m_{1_{\mathbf{R}}}\left(\left.T Y\right|_{y}\right) \leq d_{\psi} m_{\psi}\left(T Y+\eta_{+}-\left.\eta_{-}\right|_{y}\right)+d_{\psi}-1 .
$$

If the transversality condition is met, there are no obstructions to moving $\omega$ by a proper $G$-homotopy to a smooth $G$-map $h$ which is transverse to $Y$, the zero-section of $\eta_{-}$. We then set $X=h^{-1}(Y)$, $f=\left.h\right|_{X}$, and $b$ is constructed using the $G$-vector bundles $\eta_{ \pm}$. More precisely, for $H \subseteq G$, we set $X^{H}=\left(f^{H}\right)^{-1}\left(Y^{H}\right)$. Note that if a path component $X_{\alpha}^{H}$ lies in $\left(f^{H}\right)^{-1}\left(Y_{\beta}^{H}\right)$, for some component $Y_{\beta}^{H} \subseteq Y^{H}$, then $\operatorname{dim} X_{\alpha}^{H}=\operatorname{dim} Y_{\beta}^{H}$. Since $\omega$ is a $G$-fiber homotopy equivalence, we can choose the orientation of $X$ so that the $G$-map $f$ will be of degree 1.

At this point, provided that a few other conditions are met, the triple $(X, f, b)$ will be a $G$-normal map (adjusted or otherwise). However, we shall see that in our case a little additional work will render $(X, f, b)$ into an adjusted $\mathbf{D}_{2 m}$-normal map.

There is an interesting $S^{1}$-map due to Ted Petrie (see [MeP], p. 74) which will be used in our constructions. Given a pair of relatively prime integers $p$ and $q$, take integers $a$ and $b$ such that $-a p+b q=1$ and let $t^{i}$ denote the 1-dimensional complex $S^{1}$-representation where $t \in S^{1}$ acts on $\mathbf{C}$ by $t \cdot z=t^{i} z$ (complex multiplication). Define $f: t^{-2 p}+t^{-2 q}=V_{+} \rightarrow t^{-2}+t^{-2 p q}=V_{-}$by $f\left(z_{0}, z_{1}\right)=\left(\bar{z}_{0}^{a} z_{1}^{b}\right.$, $\left.z_{0}^{q}+z_{1}^{p}\right)$. It can be shown that $f$ is a proper $S^{1}$-map such that $\operatorname{deg} f^{+}=1$, where $f^{+}$is the extension of $f$ to 1 point compactifications. 
In our proof, knowledge of the structure of $L_{6}^{s}\left(\mathbf{Z}\left[\mathbf{D}_{2 m}\right], \omega\right)$, where $\mathbf{D}_{2 m}=\left\langle g, h \mid g^{m}=h^{2}=1, g h=h g^{-1}\right\rangle, w(g)=1$, and $w(h)=-1$, will be essential.

LEMMA 3.2. Assume that $m$ is such that $\widetilde{K}_{0}\left(\mathbf{Z}\left[\mathbf{D}_{2 m}\right]\right)$ has no 2-torsion. Then, $L_{6}^{s}\left(\mathbf{Z}\left[\mathbf{D}_{2 m}\right], w\right)$ decomposes into a direct sum $L_{6}^{s}\left(\mathbf{Z}\left[\mathbf{Z}_{2}\right], I D\right) \oplus \widetilde{L}\left(\mathbf{D}_{2 m}\right)$, where $\widetilde{L}\left(\mathbf{D}_{2 m}\right)$ is torsion free and ID: $\mathbf{Z}_{2} \rightarrow$ $\mathbf{Z}_{2}$ denotes the identity map. In particular, as indicated above, this result holds for primes $m \leq 67$.

Proof. First, note that the retraction and inclusion $\mathbf{D}_{2 m} \stackrel{r}{\rightarrow} \mathbf{Z}_{2} \stackrel{i}{\rightarrow}$ $\mathbf{D}_{2 m}$ are compatible with the respective orientation homomorphisms; that is,

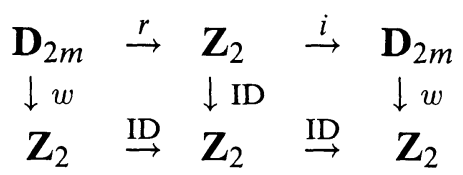

is a commutative diagram, where $r\left(g^{i} h^{\varepsilon}\right)=h^{\varepsilon} \quad(\varepsilon=0,1)$. Using the functorial properties of $L^{s}(\cdot)$ (see [W2]), it follows that $L_{6}^{s}\left(\mathbf{Z}\left[\mathbf{Z}_{2}\right]\right.$, ID) is a direct summand of $L_{6}^{s}\left(\mathbf{Z}\left[\mathbf{D}_{2 m}\right], \omega\right)$. (Note that ID: $\mathbf{Z}_{2} \rightarrow \mathbf{Z}_{2}$ is different from the trivial map $1: \mathbf{Z}_{2} \rightarrow \mathbf{Z}_{2}$.) Therefore, $L_{6}^{s}\left(\mathbf{Z}\left[\mathbf{Z}_{2}\right]\right.$, ID) is obtained by restriction. Now, according to [W3], $\widetilde{L}\left(\mathbf{D}_{2 m}\right)$ will be torsion free whenever the projective class group $\widetilde{K}_{0}\left(\mathbf{Z}\left[\mathbf{D}_{2 m}\right]\right)$ has no 2-torsion. (Also see [W1], p. 71-74. Beware of the mistakes in [W3]. They are corrected in [W1]. In these references, the computations are of $L^{\prime}(\cdot)$, the surgery obstruction group for weakly simple homotopy equivalences. However, since $\mathrm{Wh}\left(\mathbf{Z}\left[\mathbf{Z}_{2}\right]\right)=0$ and $\mathrm{Wh}\left(\mathbf{Z}\left[\mathbf{D}_{2 m}\right]\right)$ is torsion free, it turns out that for these groups and our orientation homomorphisms, $L^{\prime}(\cdot)=L^{s}(\cdot)$. This is discussed on pp. 77-78 of [W1].)

Lemma 3.2 shall be very useful to us in determining whether or not an obstruction in $L_{6}^{s}\left(\mathbf{Z}\left[\mathbf{D}_{2 m}\right], w\right)$ vanishes because nontrivial elements of $\widetilde{L}\left(\mathbf{D}_{2 m}\right)$ can be detected by the multisignature (since it's torsion free), whereas those of $L_{6}^{s}\left(\mathbf{Z}\left[\mathbf{Z}_{2}\right]\right.$, ID) are detected by the Kervaire-Arf invariant. Indeed, according to Theorem 4.14 of [W4], $L_{6}^{s}\left(\mathbf{Z}\left[\mathbf{Z}_{2}\right]\right.$, ID) is isomorphic to $\mathbf{Z}_{2}$ via the Kervaire-Arf invariant. (I [W4], $\widetilde{\mathscr{G}}$ is $L^{h}$. However, since $\mathrm{Wh}\left(\mathbf{Z}_{2}\right)=0$, we have $L_{6}^{h}\left(\mathbf{Z}\left[\mathbf{Z}_{2}\right]\right.$, ID) $=L_{6}^{s}\left(\mathbf{Z}\left[\mathbf{Z}_{2}\right]\right.$, ID).) Now, suppose the obstruction in question is associated to a normal map constructed from twice a fiber homotopy equivalence. Then since the projection from $L_{6}^{\prime}\left(\mathbf{Z}\left[\mathbf{D}_{2 m}\right], \omega\right)$ onto 
$L_{6}^{\prime}\left(\mathbf{Z}\left[\mathbf{Z}_{2}\right]\right.$, ID) is obtained by restricting the group action it follows that the Kervaire-Arf invariant of the obstruction vanishes. This follows from the fact that the Kervaire-Arf invariant can be given in terms of the Kervaire-Sullivan classes via Sullivan's characteristic variety formula (as found, for instance, on p. 152 of [BM]). The primitivity of these classes implies that a normal map obtained from twice a fiber homotopy equivalence will have vanishing Kervaire-Arf invariant.

We are now ready to handle the proof of Theorem A.

Proof of Theorem A. Let $\mathbf{D}_{2 m}$ be the dihedral group of order $2 m$, for $m$ an odd prime such that $\widetilde{K}_{0}\left(\mathbf{Z}\left[\mathbf{D}_{2 m}\right]\right)=0$ has no 2-torsion. We choose our model $Y$ to be $\mathbf{C} P^{3}$ with the following $\mathbf{D}_{2 m}$-action. If $g$ is a chosen generator of $\mathbf{Z}_{m} \subset \mathbf{D}_{2 m}$, and $z=\left[z_{0}: z_{1}: z_{2}: z_{3}\right] \in \mathbf{C} P^{3}=$ $P\left(\mathbf{C}^{4}\right)$, then $g \cdot z=\left[\xi z_{0}: \xi z_{1}: \xi^{-1} z_{2}: \xi^{-1} z_{3}\right]$, where $\xi=e^{2 \pi i / m}$. Also, we set $h \cdot z=\left[-\overline{z_{1}}: \overline{z_{0}}:-\overline{z_{3}}: \overline{z_{2}}\right]$. There are several things to notice about this action. First of all, we actually do have a $\mathbf{D}_{2 m}$-action as $g^{m} \cdot z=z, h^{2} \cdot z=\left[-z_{0}:-z_{1}:-z_{2}:-z_{3}\right]=\left[z_{0}: z_{1}: z_{2}: z_{3}\right]=z$, and $g h \cdot z=g \cdot\left[-\overline{z_{1}}: \overline{z_{0}}:-\overline{z_{3}}: \overline{z_{2}}\right]=\left[-\xi \overline{z_{1}}: \xi \overline{z_{0}}:-\xi^{-1} \overline{z_{3}}: \xi^{-1} \overline{z_{2}}\right]=$ $\left[-\overline{\xi^{-1} z_{1}}: \overline{\xi^{-1} z_{0}}:-\overline{\xi z_{3}}: \overline{\xi z_{2}}\right]=h\left[\xi^{-1} z_{0}: \xi^{-1} z_{1}: \xi z_{2}: \xi z_{3}\right]=h g^{-1} \cdot z$. It follows from an easy computation that $h$ is a free, orientation reversing involution of $Y=\mathbf{C} P^{3}$. We also have that $g^{i} h$ is free on $Y$ for $i=1, \ldots, m-1$ and therefore that $\operatorname{Iso}(Y)=\left\{1, \mathbf{Z}_{m}=\langle g\rangle\right\}$. Notice that $Y^{z_{m}}=Y_{01} \amalg Y_{23}$, where $Y_{01}=\left\{\left[z_{0}: z_{1}: 0: 0\right]: z_{0}, z_{1} \in\right.$ C $\}=\mathbf{C} P^{1}$, and $Y_{23}$ is similarly defined. Now we can proceed to the construction of an appropriate $\mathbf{D}_{2 m}$-fiber homotopy equivalence.

Let $\Sigma$ denote the sphere $S^{7}=S\left(\mathbf{C}^{4}\right)$ with the lifted maps $g$ and $h$ acting on it. Notice that these maps do not quite induce a $\mathbf{D}_{2 m^{-}}$action on $\Sigma$ because $h^{2}\left(a_{0}, a_{1}, a_{2}, a_{3}\right)=\left(-a_{0},-a_{1},-a_{2},-a_{3}\right)$, for $\left(a_{0}, a_{1}, a_{2}, a_{3}\right) \in \Sigma$. However, we do get a $\mathbf{D}_{2 m}$-action on the $V_{ \pm}-$ bundles associated to the $S^{1}$-principal bundle $\Sigma \rightarrow Y$, that is, on $\Sigma \times_{S^{1}} V_{ \pm}=\eta_{ \pm}$, where $V_{+}=t^{-2 p}+t^{-2 q}$ and $V_{-}=t^{-2}+t^{-2 p q}$ as above. (Recall that if $X$ and $Y$ are $G$-spaces, then the twisted product $X \times_{G} Y$ is the orbit space $(X \times Y) / G$, where $g(x, y)=$ $\left(x g^{-1}, g y\right)$ for all $(x, y) \in X \times Y$ and all $g \in G$.) The $\mathbf{D}_{2 m}$-action on $\Sigma \times_{S^{1}} V_{ \pm}$is given by $g[a, v]=[g a, v]$, where $[a, v]$ is the point in the orbit space corresponding to $(a, v) \in \Sigma \times V_{ \pm}$. In particular, $h^{2}[a, v]=\left[h^{2} a, v\right]=[-a, v]=[a,(-1) \cdot v]=[a, v]$. (Indeed, say $v=\left(v_{1}, v_{2}\right) \in V_{+}$. Then, $(-1) \cdot v=\left((-1)^{-2 p} v_{1},(-1)^{-2 q} v_{2}\right)=$ $\left(v_{1}, v_{2}\right)=v$.) In this way, $\eta_{ \pm}$become $\mathbf{D}_{2 m}$-vector bundles. We construct $\omega: \eta_{+} \rightarrow \eta_{-}$by taking $\operatorname{Id} \times f$, where $f: V_{+} \rightarrow V_{-}$is 
Petrie's map introduced previously, and passing to $S^{1}$-orbit spaces. Since $\operatorname{deg} f^{+}=1$, we need only check the degree condition on $\omega$ for isotropy groups $H \neq 1$. Now, the only non-trivial element of $\operatorname{Iso}(Y)$ is $\mathbf{Z}_{m}$. So, we must show that, for all $y \in Y_{01} \cup Y_{23},\left(\left.\omega\right|_{y}\right)^{\mathbf{Z}_{m}}$ has degree 1 when extended to 1 point compactifications.

At this point, we make the assumption that the relatively prime integers $p$ and $q$ are each $\equiv 1 \bmod m$. Let $\rho$ denote the 1-dimensional complex $\mathbf{Z}_{m}$-representation which sends a generator to multiplication by $e^{2 \pi i / m}$. Then, restricting the $\mathbf{D}_{2 m}$-action on $Y$ to $\mathbf{Z}_{m}$, we see that $\operatorname{res}_{\mathbf{Z}_{m}} Y=P\left(2 \rho+2 \rho^{-1}\right)$, where $P(V)$ denotes the space of complex lines in the representation $V$. From this, it follows that for $y \in Y_{01},\left.\eta_{+}\right|_{y}=\rho^{-2 q}$. Indeed, suppose $a \in \Sigma$ lies above $y$. Then $g[a, v]=[g a, v]=[\rho(g) a, v]=[a, \rho(g) v]$. Since $p$ and $q$ are each $\equiv 1 \bmod m$, we can see that, as a $\mathbf{Z}_{m}$-representation, $\left.\eta_{+}\right|_{y}=2 \rho^{-2}$. Similarly, we can compute that $\left.\eta_{-}\right|_{y}=2 \rho^{-2}$, for any $y \in Y_{01}$, and that $\left.\eta_{ \pm}\right|_{y}=2 \rho^{2}$, for any $y \in Y_{23}$. This implies that

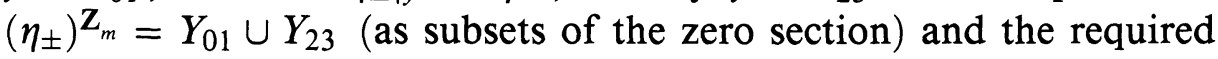
degree property easily follows. Therefore, $\omega$ is a $\mathbf{D}_{2 m}$-fiber homotopy equivalence.

It will be useful to build a $\mathbf{D}_{2 m}$-equivariant, free, orientation reversing involution into our fiber homotopy equivalence (and hence our normal map) to help with the signature obstruction which will arise later. Let $\varphi: Y \rightarrow Y$ be defined by $\varphi\left[z_{0}: z_{1}: z_{2}: z_{3}\right]=$ $\left[-\overline{z_{2}}:-\overline{z_{3}}: \overline{z_{0}}: \overline{z_{1}}\right]$ and note that $\varphi$ commutes with $\mathbf{D}_{2 m}$. Notice that $\varphi$ lifts to $\eta_{ \pm}$in such a way that $\omega$ becomes a $\mathbf{D}_{2 m} \times \mathbf{Z}_{2}$-fiber homotopy equivalence. To see this, define $\varphi^{\prime}: \Sigma \rightarrow \Sigma$ to be the obvious $\mathbf{D}_{2 m}$-map covering $\varphi$. Then $\widetilde{\varphi_{ \pm}}: \eta_{ \pm} \rightarrow \eta_{ \pm}$defined by $\widetilde{\varphi_{ \pm}}[a, v]=\left[\varphi^{\prime}(a), v\right]$ are involutions which cover $\varphi$ and make $\eta_{ \pm}$into $\mathbf{D}_{2 m} \times \mathbf{Z}_{2}$-vector bundles. It is easy to check that $\omega \circ \widetilde{\varphi_{+}}=\widetilde{\varphi_{-}} \circ \omega$ and therefore, that $\varphi$ lifts to $\eta_{ \pm}$in such a way that $\omega$ becomes a $\mathbf{D}_{2 m} \times \mathbf{Z}_{2}$-map. To see that $\omega$ is actually a $\mathbf{D}_{2 m} \times \mathbf{Z}_{2}$-fiber homotopy equivalence requires that the degree property be considered once again.

Things become a bit more complicated at this point since the addition of the involution $\varphi$ into our action introduces new elements into Iso $(Y)$. Let $H_{i}$ be the group of order two generated by $\varphi g^{j} h$, for $j=$ $0, \ldots, m-1$. Then $\operatorname{Iso}(Y)=\left\{1, \mathbf{Z}_{m}, H_{0}, \ldots, H_{m-1}\right\}$ and $Y^{H_{j}}$ $\mathbf{C} P^{1} \amalg \mathbf{C P} P^{1}$ for all $j$. To see this note that $\varphi g^{j} h\left[z_{0}: z_{1}: z_{2}: z_{3}\right] \stackrel{=}{=}$ $g^{j} \varphi h\left[z_{0}: z_{1}: z_{2}: z_{3}\right]=\left[\xi^{j} z_{3}:-\xi^{j} z_{2}: \xi^{-j} z_{1}:-\xi^{-j} z_{0}\right]$ and hence $Y^{H_{\jmath}}=\left\{\left[z_{0}: i \xi^{j} z_{1}: z_{1}: i \xi^{-j} z_{0}\right]\right\} \amalg\left\{\left[z_{0}:-i \xi^{j} z_{1}: z_{1}:-i \xi^{-j} z_{0}\right]\right\}$, where $i=\sqrt{-1}$. Notice that $Y^{H_{i}} \cap Y^{H_{j}}=\varnothing$, for all $i, j$ distinct. (One way 
to see this is: $z \in Y^{H_{i}} \cap Y^{H_{j}} \Rightarrow \varphi g^{i} h z=\varphi g^{j} h z \Rightarrow g^{i} h z=g^{j} h z \Rightarrow$ $g^{j-i} z=z \Rightarrow z \in Y_{01} \amalg Y_{23}$. But these points are not fixed by $H_{i}$ for any $i$.)

Now, take $y \in Y$ with isotropy group equal to $\left(\mathbf{D}_{2 m} \times \mathbf{Z}_{2}\right)_{y}=H_{i}=$ $\mathbf{Z}_{2}$, for some $i \in\{0, \ldots, m-1\}$. When the action on $Y$ is restricted to $H_{i}$, it is $\mathbf{Z}_{2}$-diffeomorphic to $P(1+1+h+h)$, where $h$ denotes the non-trivial 1-dimensional complex $\mathbf{Z}_{2}$-representation. (Just write the linear action of $H_{i}$ in terms of irreducible $\mathbf{Z}_{2}$-representations.) Therefore, as $H_{i}$-representations, $\left.\eta_{ \pm}\right|_{y}$ will be equivalent to $2 \cdot 1_{H_{i}}$, where $1_{H_{i}}$ denotes the 1-dimensional complex trivial $H_{i}$-representation. (Note that, for instance, if $y \in P(h+h),\left.\eta_{+}\right|_{y}=2 h^{2 p}+2 h^{-2 q}=$ $2 \cdot 1_{H_{i}}$.) Using the fact that $\operatorname{deg} f^{+}=1$, it is not hard to check that $\omega$ is indeed a $\mathbf{D}_{2 m} \times \mathbf{Z}_{2}$-fiber homotopy equivalence.

The $\mathbf{D}_{2 m} \times \mathbf{Z}_{2}$-fiber homotopy equivalence which we shall work with is $\tilde{\omega}=\omega \oplus \oplus: \widetilde{\eta_{+}}=\eta_{+} \oplus \eta_{+} \rightarrow \widetilde{\eta_{-}}=\eta_{-} \oplus \eta_{-}$. We first verify that the transversality condition holds.

We need to show that given $y \in Y$ and an irreducible $\left(\mathbf{D}_{2 m} \times \mathbf{Z}_{2}\right)_{y}$ representation $\psi$ with $m_{\psi}\left(\left.\widetilde{\eta_{-}}\right|_{y}\right) \neq 0$, we have

$$
m_{1_{\mathbf{R}}}\left(\left.T Y\right|_{y}\right) \leq d_{\psi} m_{\psi}\left(T Y+\widetilde{\eta_{+}}-\left.\widetilde{\eta_{-}}\right|_{y}\right)+d_{\psi}-1 \text {. }
$$

First, take $y \in Y$ with $\left(\mathbf{D}_{2 m} \times \mathbf{Z}_{2}\right)_{y}=H_{i}=\mathbf{Z}_{2}$, for some $i \in$ $\{1, \ldots, m-1\}$. Previous considerations show us that $\left.\left(\widetilde{\eta_{+}}-\widetilde{\eta_{-}}\right)\right|_{y}=$ $4 \cdot 1_{H_{l}}-4 \cdot 1_{H_{l}}=0$ in $R O\left(\mathbf{Z}_{2}\right)$. Also, for such $y,\left.T Y\right|_{y}=1_{H_{t}}+2 h$. Since $d_{h}=1$, we have $m_{1_{\mathbf{R}}}\left(\left.T Y\right|_{y}\right)=2 \leq m_{h}\left(T Y+\widetilde{\eta_{+}}-\left.\widetilde{\eta_{-}}\right|_{y}\right)=4$, as desired. (Recall that $m_{\psi}$ counts multiplicity as real representations.) Similarly, the desired result holds for $\psi=1_{H_{i}}$.

Next, take $y \in Y$ with $G_{y}=\mathbf{Z}_{m}$. The restricted $\mathbf{Z}_{m}$-action on $Y$ is equivalent to $P\left(2 \rho+2 \rho^{-1}\right)$, where $\rho$ is the standard $\mathbf{Z}_{m^{-}}$ representation as above. If $y \in Y_{01}$, then $\left.T Y\right|_{y}=1+2 \rho^{-2}$, while $\widetilde{\eta_{+}}-\left.\widetilde{\eta_{-}}\right|_{y}=4 \rho^{-2}-4 \rho^{-2}$, by choice of $p$ and $q$. If $y \in Y_{23}$, then $\left.T Y\right|_{y}=2 \rho^{2}+1$ and $\widetilde{\eta_{+}}-\left.\widetilde{\eta_{-}}\right|_{y}=4 \rho^{2}-4 \rho^{2}$. In either case, it is quite easy to verify the transversality condition. Of course, the stability condition holds when $y$ has trivial isotropy group.

Now that the transversality condition has been verified, we can construct the smooth $G$-manifold $X$, the $G$-map $f: X \rightarrow Y$ of degree 1, and the stable $G$-vector bundle isomorphism $b$ as indicated above.

Notice that Iso $\left(\widetilde{\eta_{+}}\right) \subseteq$ Iso $(Y)$. Indeed, due to the nature of our action, if $H$ is not in Iso $(Y)$, then it acts without fixed points on $Y$ and hence cannot be in Iso $\left(\widetilde{\eta_{+}}\right)$. This guarantees that $\operatorname{Iso}(X)=$ Iso $(Y)$. We also note that $X$ satisfies the gap hypothesis since $Y$ 
clearly does. Finally, we mention that $Y$ and all of its fixed point set components under the $G$-action are simply connected.

It follows from our construction that $X^{\mathbf{Z}_{m}}$ is $\mathbf{D}_{2 m} / \mathbf{Z}_{m}$-diffeomorphic to $Y^{Z_{m}}$. This is a result of the fact that $1_{\mathbf{Z}_{m}}$ is not a sub-representation of $\left.\widetilde{\eta_{ \pm}}\right|_{y}$ for any $y \in Y^{\mathbf{Z}_{m}}$. Now, given $H_{j} \in \operatorname{Iso}(Y)$, write $Y^{H_{j}}$ as $Y_{0}^{H_{j}} \amalg Y_{1}^{H_{j}}$. At this point, we do 0 and 1-dimensional equivariant surgeries on $X, X_{0}^{H_{J}}=\left(f^{H_{J}}\right)^{-1}\left(Y_{0}^{H_{j}}\right)$, and $X_{1}^{H_{J}}=\left(f^{H_{J}}\right)^{-1}\left(Y_{1}^{H_{j}}\right)$. This makes $X$ a connected and simply connected $\mathbf{D}_{2 m} \times \mathbf{Z}_{2}$-manifold and provides $N_{\mathbf{D}_{2 m} \times \mathbf{Z}_{2}}\left(H_{j}\right) / H_{j}=\left\langle g^{j} h\right\rangle \times \mathbf{Z}_{2}$-homotopy equivalences between $X_{i}^{H_{J}}$ and $Y_{i}^{H_{J}}$, for $i=0,1$. (Indeed, since the $X_{i}^{H_{J}}$ 's are simply connected, closed surfaces, they are 2 -spheres i.e., $\mathbf{C} P^{1}$ 's. The map $\left.f\right|_{X_{t}^{H_{J}}}$ is a map degree 1 between spheres and is hence a homotopy equivalence. It is actually a $\left\langle g^{j} h\right\rangle \times \mathbf{Z}_{2}$-homotopy equivalence since the action of this group is free.) At this stage, we forget about the $\mathbf{Z}_{2}=\{1, \varphi\}$-action, except to state that we now have an adjusted $\mathbf{D}_{2 m}$ normal map $(X, f, b)$ with target $Y$ such that the $\mathbf{D}_{2 m}$-manifold $X$ admits a $\mathbf{D}_{2 m}$-equivariant, orientation reversing involution.

We now consider the surgery obstruction $\sigma_{1}(f, b) \in L_{6}^{h}\left(\mathbf{Z}\left[\mathbf{D}_{2 m}\right], w\right)$.

Since $(X, f, b)$ is an adjusted $\mathbf{D}_{2 m}$-normal map with $f^{s}$ an equivariant diffeomorphism, we see that according to Lemmas 2.5 and 2.6, $\alpha_{G}\left(\sigma_{1}(f, b)\right)=0$. Therefore, $\sigma_{1}(f, b)$ comes from an element $\sigma_{1}^{s}(f, b) \in L_{6}^{s}\left(\mathbf{Z}\left[\mathbf{D}_{2 m}\right], w\right)$.

According to the paragraph after Lemma 3.2, the Kervaire-Arf invariant of $\sigma_{1}^{s}(f, b)$ vanishes as the $\mathbf{D}_{2 m}$-fiber homotopy equivalence $\tilde{\omega}$ is twice a fiber homotopy equivalence by construction. Therefore, $\sigma_{1}^{s}(f, b)$ must lie in the free part of $L_{6}^{s}\left(\mathbf{Z}\left[\mathbf{D}_{2 m}\right], w\right)$. (Also relevant is the formula due to Masuda [M2], which considers the Kervaire-Arf invariant for obstructions in $L(\mathbf{Z}[1], 1)$ obtained from certain fiber homotopy equivalences which are related to ours. We note that the inclusion $1 \rightarrow \mathbf{Z}_{2}$ induces an isomorphism $L(\mathbf{Z}[1], 1) \rightarrow L\left(\mathbf{Z}\left[\mathbf{Z}_{2}\right]\right.$, ID) . See p. 164 of [W2].)

Now, let $\Gamma=$ all cyclic subgroups of $\left.\mathbf{D}_{2 m}\right\}$. According to the Dress Induction Theorem found in [Dr], $L^{S}(\cdot) \otimes \mathbf{Q}$ is $\Gamma$-computable which implies that

$$
L_{6}^{s}\left(\mathbf{Z}\left[\mathbf{D}_{2 m}\right], w\right) \otimes \mathbf{Q} \stackrel{\text { Res }}{\rightarrow} \prod_{H \in \Gamma} L_{6}^{s}\left(\mathbf{Z}[H],\left.w\right|_{H}\right) \otimes \mathbf{Q}
$$

is an injection, where Res gives coordinate-wise restriction. Now, if $H$ is of order 1 or 2 , then $\operatorname{Res}_{H}\left(\sigma_{1}^{s}(f, b) \otimes \mathbf{Q}\right)=0$ because $L_{6}^{s}(\mathbf{Z}[1], 1)$ 
and $L_{6}^{s}\left(\mathbf{Z}\left[\mathbf{Z}_{2}\right]\right.$, ID) are each isomorphic to $\mathbf{Z}_{2}$ as mentioned above. That leaves us only with $\operatorname{Res}_{\mathbf{Z}_{m}}\left(\sigma_{1}^{s}(f, b) \otimes \mathbf{Q}\right) \in L_{6}^{s}\left(\mathbf{Z}\left[\mathbf{Z}_{m}\right], 1\right) \otimes \mathbf{Q}$ and this term can be shown to vanish. Indeed, let $\operatorname{Res}_{\mathbf{Z}_{m}}\left(\sigma_{1}^{s}(f, b)\right) \in$ $L_{6}^{s}\left(\mathbf{Z}\left[\mathbf{Z}_{m}\right], 1\right)$ denote the obstruction obtained by restricting the $\mathbf{D}_{2 m}$ action to $\mathbf{Z}_{m}$. Since the restricted orientation homomorphism is trivial, Petrie's formula ([P1]) applies and tells us that

$$
\operatorname{Sign}\left(\operatorname{Res}_{\mathbf{Z}_{m}}\left(\sigma_{1}^{S}(f, b)\right)\right)=\operatorname{Sign}\left(\mathbf{Z}_{m}, X\right)-\operatorname{Sign}\left(\mathbf{Z}_{m}, Y\right) .
$$

Now, by construction, $X$ admits a $\mathbf{Z}_{m}$-equivariant, orientation reversing involution and this guarantees that $\operatorname{Sign}\left(\mathbf{Z}_{m}, X\right)=0$. (Indeed, whenever a $G$-manifold $M$ admits $G$-equivariant, orientation reversing diffeomorphism, we have that $\operatorname{Sign}(G, M)=0$.) Since $\operatorname{Sign}\left(Z_{m}, Y\right)$ is obviously 0 , we have that $\operatorname{Sign}\left(\operatorname{Res}_{\mathbf{z}_{m}}\left(\sigma_{1}^{s}(f, b)\right)\right)=0$. But this implies that $\operatorname{Res}_{\mathbf{Z}_{m}}\left(\sigma_{1}^{S}(f, b) \otimes \mathbf{Q}\right)$ vanishes because the torsion free part of $L_{6}^{s}\left(\mathbf{Z}\left[\mathbf{Z}_{m}\right], 1\right)$ is detected by the multisignature. Hence, by the injectivity of Res, we have that $\sigma_{1}^{S}(f, b) \otimes \mathbf{Q}=0$. This means that $\sigma_{1}^{s}(f, b)$ lies in the torsion part of $L_{6}^{s}\left(\mathbf{Z}_{2}\left[\mathbf{D}_{2 m}\right], w\right)$ and as we saw this implies that $\sigma_{1}^{s}(f, b)=0$. Thus, $\sigma_{1}(f, b)=0$ in $L_{6}^{h}\left(\mathbf{Z}\left[\mathbf{D}_{2 m}\right], w\right)$ and we can use $\mathbf{D}_{2 m}$-surgery to construct the triple $\left(X_{\tilde{\eta}}, F, B\right)$, where $F: X_{\tilde{\eta}} \rightarrow Y=\mathbf{C} P^{3}$ is a $\mathbf{D}_{2 m}$-homotopy equivalence.

The stable $G$-vector bundle isomorphism $B$ between $T X_{\tilde{\eta}}$ and $F^{*}\left(T Y+\widetilde{\eta_{+}}-\widetilde{\eta_{-}}\right)$allows us to compute the Pontryagin class of the smooth manifold $X_{\tilde{\eta}}$. In particular, the first Pontryagin class is given by $p_{1}\left(X_{\tilde{\eta}}\right)=\left(4+8\left(p^{2}-1\right)\left(q^{2}-1\right)\right) x^{2}$, where $x \in H^{2}\left(X_{\tilde{\eta}}\right)$ is a generator. (See [H] $\S \S 6$ and 7 for more details on this calculation.) So, $X_{\tilde{\eta}}=X_{k}$, where $k=\left(p^{2}-1\right)\left(q^{2}-1\right) / 3$ and by varying $p$ and $q$ (within the constraints that $p$ and $q$ are relatively prime and each $\equiv 1 \bmod m$ ) we can build $\mathbf{D}_{2 m}$-actions for infinitely many $k$.

\section{REFERENCES}

[AS] M. F. Atiyah and I. M. Singer, The index of elliptic operators III, Annals of Math., 87 (1968), 546-604.

[B] G. Bredon, Equivariant Cohomology Theories, Springer Lecture Notes in Math., no. 34 (1967).

[BM] G. Brumfiel and I. Madsen, Evaluation of the transfer and the universal surgery classes, Invent. Math., 32 (1976), 133-169.

[BQ] W. Browder and F. Quinn, A Surgery Theory for $G$ Manifolds and Stratified Sets, Manifolds-Tokyo, University of Tokyo Press, (1973), 27-36.

[D] K. H. Dovermann, Almost isovariant normal maps, Amer. J. Math., 111, no. 6, (Dec. 1989), 851-904.

[DM] K. H. Dovermann and M. Masuda, Exotic cyclic actions on homotopy complex projective spaces, J. Fac. Sci. Univ. Tokyo Sect. IA Math. 37, No. 2, 335-376. 
[DMS] K. H. Dovermann, M. Masuda, and R. Schultz, Conjugation involutions on homotopy complex projective spaces, Japanese J. Math., 12 (1986), 1-35.

[DP] K. H. Dovermann and T. Petrie, G-Surgery II, Mem. Amer. Math. Soc., 260 (1982),

[DR1] K. H. Dovermann and M. Rothenberg, The Generalized Whitehead Torsion of a G-Fiber Homotopy Equivalence, Transformation Groups, Proc. Conf. Osaka Japan 1987, Springer LNM, 1375 (1989), 60-88.

[DR2] __ Equivariant surgery and classification of finite group actions on manifolds, Mem. Amer. Math. Soc., 397 (1988),

[Dr] A. Dress, Induction and structure theorems for orthogonal representations of finite groups, Annals of Math., 102 (1975), 291-325.

[H] M. Hughes, Finite group actions on homotopy complex projective spaces, Math. Zeit., Band 199, Heft 1, (1988), 133-151.

[L] M. P. Lee, Integral representations of dihedral groups of order $2 p$, Trans. Amer. Math. Soc., 110 (1964), 213-231.

[M1] M. Masuda, Smooth involutions on homotopy $\mathbf{C} P^{3}$, Amer. J. Math., 106 (1984), 1487-1501.

[M2] _ The Kervaire invariant of some fiber homotopy equivalences, Adv. Studies in Pure Math. vol. 9, Kinokuniya North Holland.

[Ma] B. Magurn, $S K_{1}$ of dihedral groups, J. Algebra, 51 (1978), 399-415.

[MeP] A. Meyerhoff and T. Petrie, Quasi-equivalence of G-modules, Topology, 15 (1976), 69-75.

[Mo] M. Morimoto, Bak groups and equivariant surgery, $K$-Theory, 2, no. 4, (1989), 465-483.

[MY] D. Montgomery and C. T. Yang, Differentiable actions on homotopy seven spheres I, Trans. Amer. Math. Soc., 112 (1966) 480-498.

[O] T. Obayashi, On the Whitehead group of the dihedral group of order $2 p$, Osaka J. Math., 8 (1971), 291-297.

[P1] T. Petrie, The Atiyah-Singer invariant, the Wall groups $L_{n}(\pi, 1)$ and the function $t e^{x}+1 / t e^{x}-2$, Annals of Math., 92 (1970), 174-187.

[P2] _ Pseudo-equivalences of G-manifolds, Proc. Symp. Pure Math., 32 (1978), 169-210.

[PR] T. Petrie and J. Randall, Transformation Groups on Manifolds, Dekker Lecture Series, Marcel Dekker Inc., 48 (1984).

[Sh] J. Shaneson, Wall's surgery obstruction groups for $G \times \mathbf{Z}$, Annals of Math., 90 (1969), 296-334.

[W1] C. T. C. Wall, Classification of Hermitian forms VI, Annals of Math., 103 (1976), 1-80.

[W2] _ Surgery on Compact Manifolds, Academic Press, 1970.

[W3] _ Some L groups of finite groups, Bull. Amer. Math. Soc., 79 (May 1973), 526-529.

[W4] _ _ Surgery of non-simply connected manifolds, Annals of Math., 84 (1966), 217-276.

[Wa] L. C. Washington, Introduction to Cyclotomic Fields, Graduate Texts in Math., 83, Springer-Verlag, New York, 1982

Received May 8, 1989 and in revised form September 10, 1990.

Florida State University

TALlahasseE, FL 32306-3027 


\section{PACIFIC JOURNAL OF MATHEMATICS EDITORS}

\author{
V. S. VARADARAJAN \\ (Managing Editor) \\ -University of California \\ Los Angeles, CA 90024-1555-05 \\ Herbert Clemens \\ University of Utah \\ Salt Lake City, UT 84112 \\ THOMAS ENRIGHT \\ University of California, San Diego \\ La Jolla, CA 92093
}

R. FINN

Stanford University

Stanford, CA 94305

Hermann FlaschKa

University of Arizona

Tucson, AZ 85721

VAUGHAN F. R. JONES

University of California

Berkeley, CA 94720

SteVen KerCKhoff

Stanford University

Stanford, CA 94305
C. C. MOORE

University of California

Berkeley, CA 94720

Martin ScharlemanN

University of California

Santa Barbara, CA 93106

\section{HAROLD STARK}

University of California, San Diego

La Jolla, CA 92093

\section{ASSOCIATE EDITORS}

\begin{tabular}{|c|c|c|c|c|}
\hline R. Arens & $\begin{array}{l}\text { E. F. BECKENBACH } \\
(1906-1982)\end{array}$ & Neumann & $\begin{array}{l}\text { F. WolF } \\
(1904-1989)\end{array}$ & K. Yoshida \\
\hline \multicolumn{5}{|c|}{ SUPPORTING INSTITUTIONS } \\
\hline \multicolumn{2}{|c|}{ UNIVERSITY OF ARIZONA } & \multicolumn{3}{|c|}{ UNIVERSITY OF OREGON } \\
\hline \multirow{2}{*}{\multicolumn{2}{|c|}{$\begin{array}{l}\text { UNIVERSITY OF BRITISH COLUMBIA } \\
\text { CALIFORNIA INSTITUTE OF TECHNOLOGY }\end{array}$}} & \multicolumn{3}{|c|}{ UNIVERSITY OF SOUTHERN CALIFORNIA } \\
\hline & & \multicolumn{3}{|c|}{ STANFORD UNIVERSITY } \\
\hline CALIFORNIA INSTITUTE OF TECHNOLOGY & UNIVERSITY OF CALIFORNIA & \multicolumn{3}{|c|}{ UNIVERSITY OF HAWAII } \\
\hline \multicolumn{2}{|c|}{ MONTANA STATE UNIVERSITY } & \multicolumn{3}{|c|}{ UNIVERSITY OF TOKYO } \\
\hline \multicolumn{2}{|c|}{ UNIVERSITY OF NEVADA, RENO } & \multicolumn{3}{|c|}{ UNIVERSITY OF UTAH } \\
\hline \multicolumn{2}{|c|}{ NEW MEXICO STATE UNIVERSITY } & \multicolumn{3}{|c|}{ WASHINGTON STATE UNIVERSITY } \\
\hline OREGON STA & UNIVERSITY & UNIVERS & OF WASHING & \\
\hline
\end{tabular}




\section{Pacific Journal of Mathematics}

Vol. 150, No. $1 \quad$ September, 1991

Jan Aarts, Charles Lemuel Hagopian and Lex Gerard Oversteegen, The orientability of matchbox manifolds $\ldots \ldots \ldots \ldots \ldots \ldots \ldots \ldots \ldots \ldots \ldots \ldots$

Roger W. Barnard, Carl Hanson Fitzgerald and Sheng Gong, The growth and $1 / 4$-theorems for starlike mappings in $\mathbf{C}^{n} \ldots \ldots \ldots \ldots \ldots \ldots \ldots$

Allan Berele, Trace rings for verbally prime algebras $\ldots \ldots \ldots \ldots \ldots \ldots \ldots 23$

Quo-Shin Chi, Curvature characterization and classification of rank-one

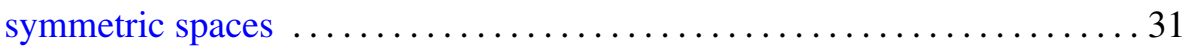

Harold Gerard Donnelly and Jeffrey Marc Lee, Domains in Riemannian

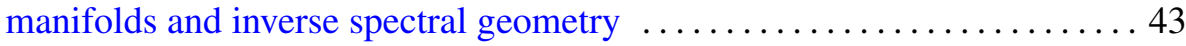

Clifford John Earle, Jr. and Patricia Lilaine Sipe, Families of Riemann

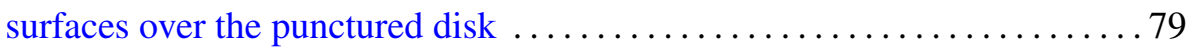

Mark P. Hughes, Dihedral group actions on homotopy complex projective

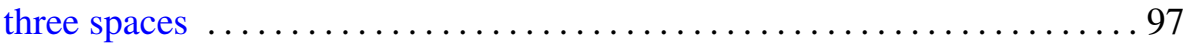

Robert F. Lax and Carl Widland, Gap sequences at a singularity ........111

Takahiko Nakazi, Bounded Hankel forms with weighted norms and lifting

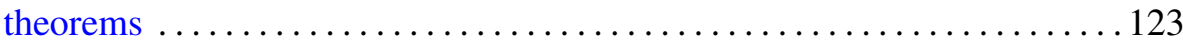

Douglas Murray Pickrell, Mackey analysis of infinite classical motion groups

Martha Rzedowski-Calderón and Gabriel Daniel Villa-Salvador,

Automorphisms of congruence function fields 167

Peter N-S Wong, Equivariant Nielsen fixed point theory for $G$-maps 